\title{
Regularization in ejection-collision orbits of the RTBP
}

\author{
Mercè Ollé, Òscar Rodríguez and Jaume Soler
}

\begin{abstract}
Numerical explorations confirm well-known analytical results on the existence of ejection-collision orbits in the restricted three-body problem for very restrictive values of the Jacobi constant $C$. For different values of $C$ some new types of ejection-collision orbits are found. The concept of $n$-ejection-collision orbit is introduced and numerical explorations are carried out which show a very rich dynamics when Hill regions contain both primaries. Complete details on the numerical methods and the bifurcations of the different families of orbits are given in the references.
\end{abstract}

\section{Introduction}

The object of this piece of work is to contribute with some results on ejectioncollision (EC) orbits in the restricted three-body problem (RTBP). Orbits which eject from or collide with one of the primaries are of particular interest because they are relevant to astronomical problems such as the determination of regions of capture of irregular moons by giant planets (see [1]) or explaining the formation of Kuiperbelt binaries by means of physical collisions between the binary and intruders (see [2]). They are also relevant for some microscopic scale problems. The study of the hydrogen atom subject to a circularly polarised microwave field, where the collisions between the electron and the core play an important role to explain ionisation, is an instance of such an application (see [5] and [15]).

Mercè Ollé

Dept. de Matemàtiques, Universitat Politècnica de Catalunya. e-mail: merce.olle@upc.edu

Òscar Rodríguez

Dept. de Matemàtiques, Universitat Politècnica de Catalunya. e-mail: oscar.rodriguez@upc.edu

Jaume Soler

Dept. d'Enginyeria Civil i Ambiental, Universitat Politècnica de Catalunya.

e-mail: jaume.soler.villanueva@upc.edu 
Concerning analytical studies of EC orbits in the RTBP (planar, spatial, circular and elliptic cases), a perturbation approach is usually considered and the McGehee regularization ([14]) is typically used. We emphasise that in all the papers published so far, the EC orbits analysed are what below we will call 1-EC orbits, i.e. orbits that eject from the primary, reach one maximum distance and come back to collision with the same primary. The references to be mentioned are: (i) in the planar RTBP, Llibre [11] (existence of at least two EC orbits for the mass parameter $\mu>0$ small enough and the energy $H$ small enough), Lacomba and Llibre [10] (by means of the existence of transversal EC orbits the authors prove that both the Hill problem and the RTBP have no $C^{1}$-extensible regular integrals), and Chenciner and Llibre [6] (existence of four EC orbits for any value of $\mu \in(0,0.5]$ and $H$ small enough). (ii) In the spatial RTBP, Llibre and Martinez Alfaro [12] (existence of EC orbits for small enough values of the mass parameter). (iii) In the planar elliptic RTBP, Llibre and Pinyol [13] and Pinyol [17] (existence of EC orbits for both the mass parameter and the eccentricity small enough). It is our purpose to prove the existence of n-EC orbits, $n>1$, i.e. ejection orbits that reach $n$ maxima in the distance before going back to collision. This will appear in a future paper.

Focussing on numerical results, there are some isolated computations published: we mention Henon's paper (see [8]) about the computation of EC orbits obtained along the continuation of some families of symmetric periodic -non-collision- orbits in the Copenhagen problem (that is $\mu=0.5$ ) and also for Hill's problem (see [9]). Finally, the evolution of 16 particular collision periodic orbits obtained from the $\mu=0.5$ case was numerically studied for various values of the mass ratio $\mu$ in [4].

The present piece of work has two main goals. First, it summarises the results put forward by the authors in [16]: (i) the existence of only four 1-EC orbits for any value of $\mu>0$ and very small values of $H$ (known analytical results) is confirmed and this result is numerically extended to less restrictive values of the energy. For higher values of the energy the Hill region contains the other primary and some new EC orbits appear. On the other hand, the concept of a family of $n$-EC orbits is introduced and some bifurcations along these families appear. The reader is referred to [16] for complete details on the numerical methods and the description of the bifurcations of the different families of orbits (not described here). We emphasize that in the mentioned paper only the McGehee regularization was taken into account and therefore each EC orbit is regarded as a heteroclinic orbit, so the time from ejection to collision is infinity. However, in the present work, we will also consider the Levi-Civita regularization and we will comment on the pros and cons when comparing both regularizations. This is precisely the second goal of this chapter.

\section{Ejection-collision in the RTBP}

In this section, (i) we recall some properties of the RTBP, (ii) we introduce two regularizations, (iii) we analyse the collision manifold, and (iv) we present some results for $n$-EC orbits, $n \geq 1$. 


\subsection{The RTBP}

We use the standard setting of the planar restricted three-body problem (RTBP): the primaries of masses $m_{1}=1-\mu$ and $m_{2}=\mu$ occupy, respectively, the positions $(-\mu, 0)$ and $(1-\mu, 0)$ on the $x$-axis of a rotating frame (the synodical frame). With these assumptions, the equations of motion for the particle in this rotating are given by

$$
\begin{aligned}
\ddot{x}-2 \dot{y} & =\frac{\partial \Omega}{\partial x}(x, y) \\
\ddot{y}+2 \dot{x} & =\frac{\partial \Omega}{\partial y}(x, y),
\end{aligned}
$$

where ${ }^{\cdot}=d / d t$ and

$$
\Omega(x, y)=\frac{1}{2}\left(x^{2}+y^{2}\right)+\frac{1-\mu}{\sqrt{(x+\mu)^{2}+y^{2}}}+\frac{\mu}{\sqrt{(x+\mu-1)^{2}+y^{2}}}+\frac{1}{2} \mu(1-\mu) .
$$

It is well known that this system of ODE has the following properties (see [21] for details).

1. There exists a first integral, the Jacobi integral, given by

$$
C=2 \Omega(x, y)-\dot{x}^{2}-\dot{y}^{2} .
$$

2. The equations of motion are invariant under the symmetry

$$
(t, x, y, \dot{x}, \dot{y}) \longrightarrow(-t, x,-y,-\dot{x}, \dot{y})
$$

which translates into the well-known symmetry of the orbits.

3. There exist five equilibrium points: the collinear ones, $L_{i}, i=1,2,3$ on the $x$ axis, and the triangular ones $L_{i}, i=4,5$ located at the vertices of an equilateral triangle with the primaries. We denote by $x_{L_{i}}$ the abscissa of point $L_{i}$. We assume $\mu l e 1 / 2$ and $x_{L_{2}} \geq 1-\mu \geq x_{L_{1}} \geq-\mu \geq x_{L_{3}}$, so that $L_{1}$ is between the primaries, $L_{2}$ is on the right of the small one and $L_{3}$ on the left of the large one. We denote by $C_{L_{i}}(\mu)$ the value of the Jacobi constant at $L_{i}$ for a given $\mu$.

4. The equations of motion can be written as a Hamiltonian system in the coordinates $(x, y)$ and associated momenta $\left(p_{x}, p_{y}\right)$. The Hamiltonian function is

$$
H\left(x, y, p_{x}, p_{y}\right)=\frac{1}{2}\left(p_{x}^{2}+p_{y}^{2}\right)+y p_{x}-x p_{y}-\frac{1-\mu}{r_{1}}-\frac{\mu}{r_{2}}-\frac{1}{2} \mu(1-\mu),
$$

with $r_{1}=\sqrt{(x+\mu)^{2}+y^{2}}$ and $r_{2}=\sqrt{(x+\mu-1)^{2}+y^{2}}$, and the relation between $C$ and $H$ is given by

$$
H=-\frac{C}{2} \text {. }
$$

We denote by $H_{L_{i}}(\mu)$, the associated value of the Hamiltonian at $L_{i}$ for a given $\mu$. 
5. Given a value of the Jacobi constant $C$ (or the Hamiltonian $H$ ), the motion is allowed to take place only in the Hill region defined by

$$
\mathcal{R}(C)=\left\{(x, y) \in \mathbb{R}^{2} \mid 2 \Omega(x, y) \geq C\right\} .
$$

In this paper we will restrict the values of $C$ to the range $C \geq C_{L_{2}}(\mu)$ (equivalently $H \leq H_{L_{2}}(\mu)$, see in Figure 1 the corresponding Hill regions). More precisely, we first study the existence of EC orbits with the big primary for $C \geq C_{L_{1}}(\mu)$ (see Figure 1 bottom), where only the bounded region around the big primary is taken into account. Later on, we consider also $C \geq C_{L_{2}}(\mu)$, where the motion can take place in a bounded region containing both primaries, and therefore, there also exist orbits that eject from one primary and collide with the other one. Actually, the dynamics is very rich because of the Lyapunov periodic orbits around $L_{1}$ and their associated invariant manifolds. Specific values of $H$ can be translated to values of $C$ through the relation (5).
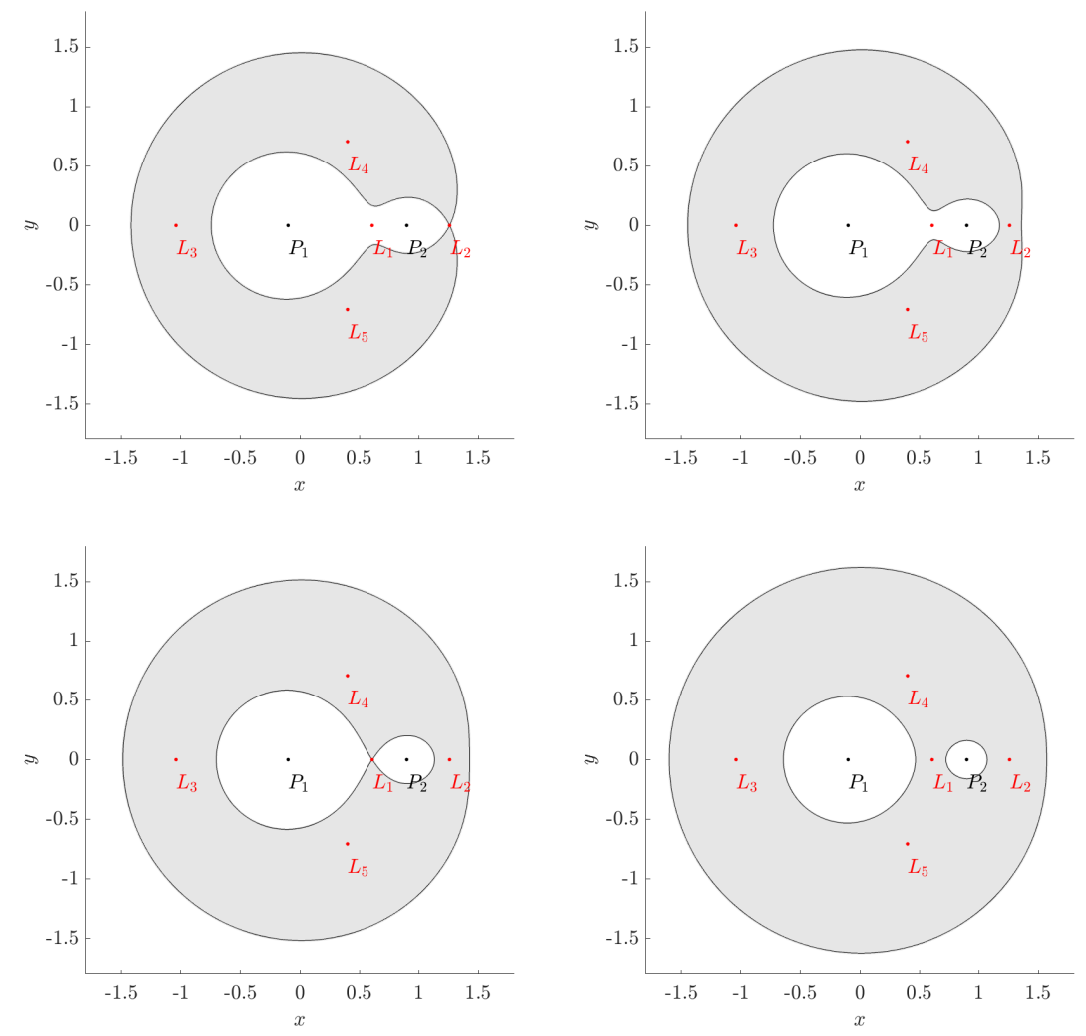

Fig. 1 Hill's region according to the Jacobi constant $C$. Top left: $C=C_{L_{2}}$; top right: $C_{L_{2}}<C<$ $C_{L_{1}}$; bottom left: $C=C_{L_{1}}$; bottom right: $C>C_{L_{1}}$ 


\subsection{Two regularizations}

As the objective is to study the ejection-collision orbits of the big primary, we will deal with the singularity $r_{1}=0$ in the equations introducing two types of regularizations: following the work of McGehee (see [14], [7]) and Levi-Civita coordinates (see [21]).

\section{McGehee regularization}

We apply the translation $q_{1}=x+\mu, q_{2}=y$, to locate the primary of mass $1-\mu$ at) the origin of coordinates and that of mass $\mu$ at $(1,0)$ and we introduce the canonical change of polar coordinates

$$
\begin{array}{ll}
q_{1}=r \cos \theta & p_{1}=p_{r} \cos \theta-\frac{p_{\theta}}{r} \sin \theta \\
q_{2}=r \sin \theta & p_{2}=p_{r} \sin \theta+\frac{p_{\theta}}{r} \cos \theta
\end{array}
$$

which changes the Hamiltonian into

$$
\begin{aligned}
H\left(r, \theta, p_{r}, p_{\theta}\right)= & \frac{1}{2}\left(p_{r}^{2}+\frac{p_{\theta}^{2}}{r^{2}}\right)-p_{\theta}-\frac{1-\mu}{r}+\mu\left(p_{r} \sin \theta+\frac{p_{\theta}}{r} \cos \theta\right) \\
& -\frac{\mu}{\sqrt{1+r^{2}-2 r \cos \theta}}-\frac{1}{2} \mu(1-\mu)
\end{aligned}
$$

Then we introduce the new variables

$$
v=\dot{r} r^{1 / 2} \quad u=r^{3 / 2} \dot{\theta}
$$

and a change of time $d t / d \tau=r^{3 / 2}$, such that the system of ODE becomes

$$
\begin{aligned}
r^{\prime}= & v r \\
\theta^{\prime}= & u \\
v^{\prime}= & \frac{1}{2} v^{2}+u^{2}+2 u r^{3 / 2}+r^{3}-(1-\mu) \\
& \quad-\mu r^{2} \cos \theta-\mu r^{2} \frac{r-\cos \theta}{\left(1+r^{2}-2 r \cos \theta\right)^{3 / 2}} \\
u^{\prime}= & -\frac{1}{2} u v-2 v r^{3 / 2}+\mu r^{2} \sin \theta\left(1-\frac{1}{\left(1+r^{2}-2 r \cos \theta\right)^{3 / 2}}\right),
\end{aligned}
$$

where $^{\prime}=d / d \tau$. 


\section{Levi-Civita regularization}

The Levi-Civita regularization consists of the transformation (see Fig. 2) given by

$$
\left\{\begin{array}{l}
x=-\mu+u^{2}-v^{2} \\
y=2 u v \\
\frac{d t}{d s}=4\left(u^{2}+v^{2}\right) .
\end{array}\right.
$$
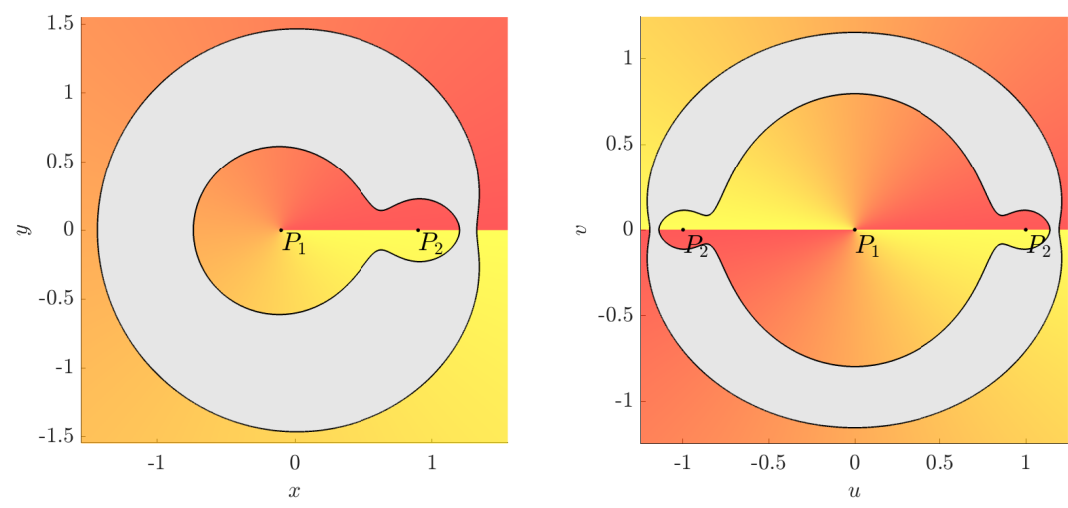

Fig. 2 Levi-Civita transformation for $\mu=0.1$. Left. $(x, y)$ variables. Right. Levi-Civita ones $(u, v)$. In grey the forbidden Hill's region for $C=3.58$.

Under this transformation the system (1) becomes (see details in [21]):

$$
\left\{\begin{array}{l}
u^{\prime \prime}-8\left(u^{2}+v^{2}\right) v^{\prime}=\left(4 U\left(u^{2}+v^{2}\right)\right)_{u} \\
v^{\prime \prime}+8\left(u^{2}+v^{2}\right) u^{\prime}=\left(4 U\left(u^{2}+v^{2}\right)\right)_{v}
\end{array}\right.
$$

where $^{\prime}=d / d s$ and

$$
U=\frac{1}{2}\left[(1-\mu)\left(u^{2}+v^{2}\right)^{2}+\mu r_{1}^{2}\right]+\frac{1-\mu}{u^{2}+v^{2}}+\frac{\mu}{r_{1}}-\frac{C}{2} .
$$

with $r_{1}=\sqrt{\left(-1+u^{2}-v^{2}\right)^{2}+4 u^{2} v^{2}}$. 


\subsection{The collision manifold}

Two advantages in using McGehee regularization are that the system of ODE in these variables is simpler, and that we have the so-called collision manifold that describes both the motion at the ejection/collision (by means of a blow-up) and it gives insight into the motion close to ejection/collision.

System (8) has an invariant manifold $\Lambda$ defined by $r=0$, called the collision manifold. This manifold $\Lambda$ is a torus (see Figure 3 ) given by

$$
\Lambda=\left\{u^{2}+v^{2}=2(1-\mu), \quad \theta \in[0,2 \pi]\right\}
$$

and the dynamics on this torus is governed by the equations

$$
\begin{aligned}
\theta^{\prime} & =u \\
v^{\prime} & =\frac{1}{2} v^{2}+u^{2}-(1-\mu) \\
u^{\prime} & =-\frac{1}{2} u v .
\end{aligned}
$$

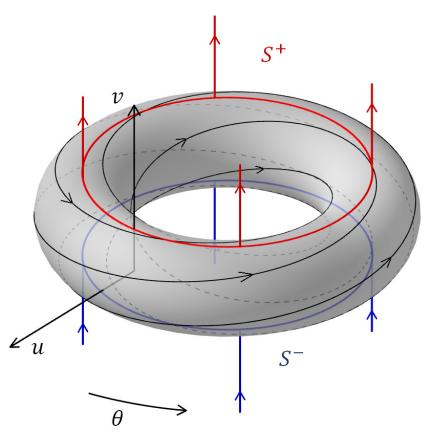

Fig. 3 The collision manifold

There exist two circles of equilibrium points on $\Lambda$ defined by $S^{+}=\{r=0, \theta, v=$ $\left.v_{0}, u=0, \theta \in[0,2 \pi]\right\}$ and $S^{-}=\left\{r=0, \theta, v=-v_{0}, u=0, \theta \in[0,2 \pi]\right\}$ with $v_{0}=+\sqrt{2(1-\mu)}$.

For a value of the Jacobi constant fixed, each equilibrium point $P \in S^{+}$has a 1-d unstable manifold $W^{u}(P)$ and a 1-d stable one $W^{s}(P)$. Similarly, each equilibrium point $Q \in S^{-}$, has a 1-d stable manifold $W^{s}(Q)$ and a 1-d unstable one $W^{u}(Q)$. In Figure $3, W^{u}(P)$ and $W^{s}(Q)$ are symbolically represented by red and blue arrows respectively.

We distinguish 3 types of orbits: (i) ejection, (ii) collision and (iii) ejectioncollision orbits. 
(i) The set of ejection orbits - those which are ejected from collision with the big primary - is the set of orbits on the unstable manifold $W^{u}(P)$, for any $P=$ $\left(0, \theta, v_{0}, 0\right) \in S^{+}$. So each ejection orbit may be regarded as an orbit such that $r>0$ for all finite time $\tau$ and asymptotically tends to an equilibrium point $P \in S^{+}$as $\tau \rightarrow-\infty$.

(ii) The set of collision orbits - those which arrive at collision with the big primary - is the set of orbits on the stable manifold $W^{s}(Q)$, for any $Q=$ $\left(0, \theta,-v_{0}, 0\right) \in S^{-}$. So each collision orbit may be regarded as an orbit such that $r>0$ for all finite time $\tau$ and asymptotically tends to an equilibrium point $Q \in S^{-}$ as $\tau \rightarrow+\infty$.

(iii) The set of ejection-collision orbits - those which eject from the big primary and then collide with it - is the set of orbits obtained from the intersection $W^{u}\left(S^{+}\right) \cap$ $W^{s}\left(S^{-}\right)$. So they may be regarded as heteroclinic orbits between $P \in S^{+}$and $Q \in S^{-}$.

Finally we define $n$-ejection-collision orbits, simply denoted by $n$-EC orbits, as those orbits that eject from the big primary, reach $n$ times a relative maximum of the distance $r$, with $n-1$ close approaches in between, before colliding with the big primary.

At this point it is worthwhile to compare the two regularizations contemplated in this chapter (McGehee and Levi-Civita) when applied to the study of ejection/collision orbits. When we consider the Levi-Civita regularization, ejection/collision orbits are simply orbits that leave from/arrive at the origin, which is now a regular point, so it takes a finite range of time to describe an EC orbit and we do not have the collision manifold. By contrast, it takes an infinite time to describe an EC orbit in McGehee coordinates, since they are asymptotic (heteroclinic) connections. From this point of view, although the system of ODE in Levi-Civita variables is more intricated, the numerical computations are really faster. Moreover, the initial conditions of an ejection orbit are on invariant manifolds of equilibrium points when using McGehee variables, whereas in Levi-Civita variables, we simply take the set

$$
u=v=0, \quad u^{\prime}=\sqrt{8(1-\mu)} \cos \theta, \quad v^{\prime}=\sqrt{8(1-\mu)} \sin \theta, \quad \theta \in[0,2 \pi]
$$

A simple numerical method to detect EC orbits in McGehee variables can be implemented: we take a set of initial conditions on $W^{u}\left(S^{+}\right)$, integrate forward in time up to the $2 n$-th crossing with the Poincaré section $v=0$ and detect singularities in time due to the asymptotic behaviour which characterises EC orbits (see [16] for details).

When using Levi-Civita variables, we integrate the set of initial conditions (13) up to the $n$-th crossing with the Poincare section $r=r_{\text {max }}$ and obtain a curve on this section. Then we proceed the same way backwards in time, obtaining another curve (this last task is not actually computed due to the symmetry (4). The intersection points between both curves belong to EC-orbits.

In summary, McGehee regularization applied to the study of ejection/collision orbits requires integrating for a long time, taking initial conditions on the invariant manifolds -and this means that such initial conditions will be approximated but not 
on the invariant manifold itself-, and dealing with the fact that successive passages through collision are very badly conditioned because of the impossibility to reach an infinite time. On the other hand, with Levi-Civita regularization only finite shorter ranges of time are required, the passage through collision is a regular point and the initial conditions are exact and there is no problem at all to consider integration spans with successive collisions. So, from the numerical point of view, Levi-Civita is really preferable.
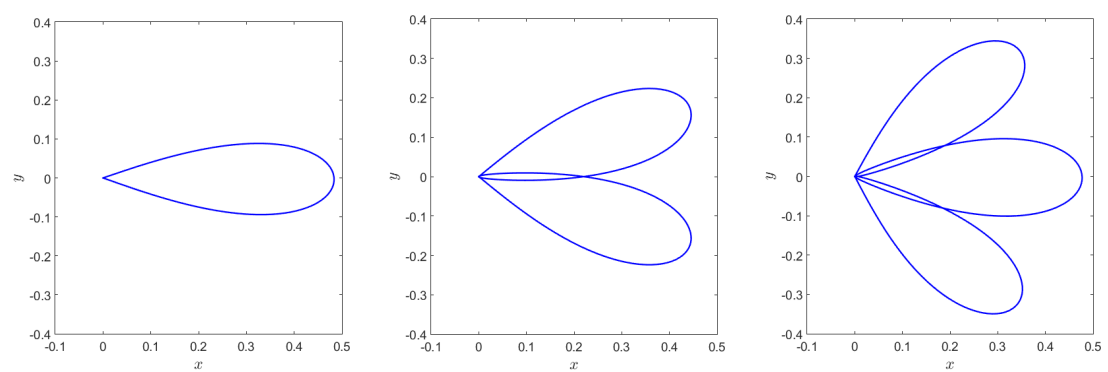

Fig. 4 Examples of $n$-ejection-collision orbits for $n=1,2,3$ (from left to right). For $n=2(n=3)$, there are 1 (2) close passages to collision between ejection and collision.

\subsection{Results for 1-EC orbits}

\subsubsection{Existence of four 1-EC orbits.}

Although the analytical papers concerning the existence of 1-EC orbits typically consider very restrictive values of $H$ and small values of $\mu$, we have done extensive numerical explorations on a grid of values of $\mu$ in the interval [0.01, 0.5] and $\theta_{0} \in$ $[0,2 \pi]$ for $H \leq H_{L_{1}}(\mu)$. The simulations done confirm the existence of only four ejection-collision orbits. See in Figure 4 examples of $n$-EC orbits for $n=1,2,3$. In Figure 5 left, we plot the four 4-EC orbits existing for particular values of $H$ and $\mu=0.5$.

Figure 5 shows the curves $W^{u}\left(S^{+}\right) \cap \Sigma_{1}$ and $W^{s}\left(S^{-}\right) \cap \Sigma_{1}$ (where $\Sigma_{1}$ denotes the first intersection with the Poincare section $v=0$ ) for $\mu=0.5$ (for other values of $\mu$ see [18]) and different values of $H$ (Figure 5 left). Also shown (in black) are the corresponding 1-EC orbits on the $(x, y)$-plane.

The existence of only four 1-EC orbits is no longer true for higher values of the energy $H$, since new ones show up. We refer the reader to the paper [16] for the details and the description of appearing bifurcations. 

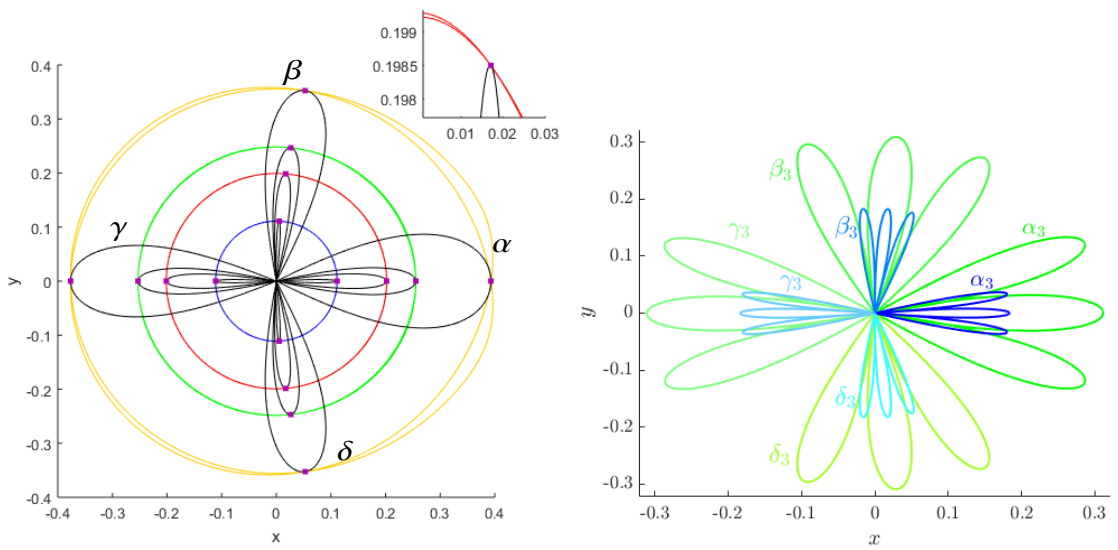

Fig. 5 Left. $\mu=0.5, W^{u}\left(S^{+}\right) \cap \Sigma_{1}$ and $W^{s}\left(S^{-}\right) \cap \Sigma_{1}$ for values of $H-5.25$ in blue, -3.25 in red, -2.75 in green and $H_{L_{1}}(\mu)$ in yellow. In black the 1-EC orbits for such values of $H$. In purple the points of the EC orbits at $\Sigma_{1}$ and in black the projections of the EC orbits on the configuration plane $(x, y)$. Right. The four $n$-ejection-collision orbits for $\mu=0.1$ (left) and $n=3$ for $H=-5.05$ (blue) and $H=-3.05$ (green).

\subsubsection{Results for $n$-EC orbits}

We have also done extensive numerical simulations on a grid of values for $\mu \epsilon$ $[0.01,0.5]$ and energy levels $H<H_{L_{1}}(\mu)$ and we can conclude that for all $n$ there exists a value $\hat{H}(\mu, n)$ such that for $H \leq \hat{H}(\mu, n)$ there are four $n$-ejection-collision orbits, which can be characterised in a way similar to the characterization of the 1-ejection-collision orbits. For example, see the four 3-EC orbits for $\mu=0.1$ and different values of $H$ in Figure 5 right.

Further details on the bifurcations of $n$-EC orbits and the numerical methods can be found in [16].

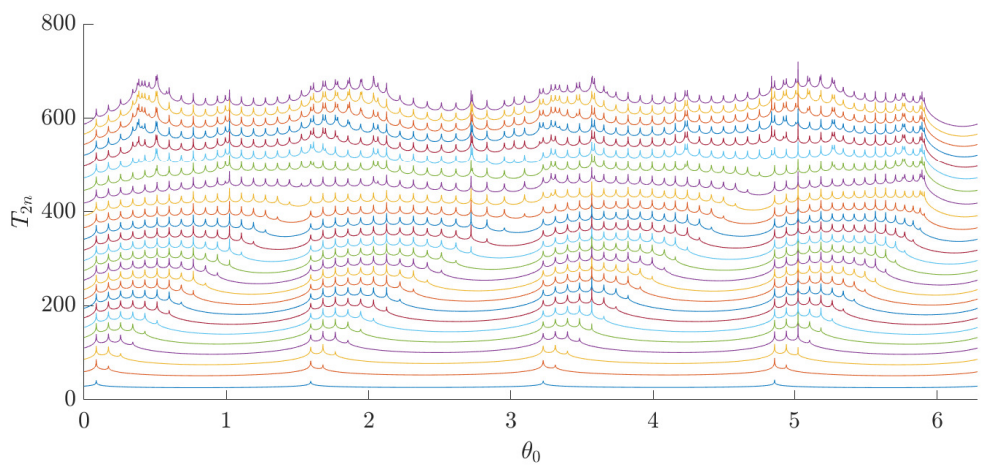

Fig. $6\left(\theta_{0}, T_{2 n}\right), n=1, \ldots, 25$ for $\mu=0.5$ and $H=-4$. 
We remark that for high values of $n$, applying McGehee or Levi-Civita really makes a difference: using McGehee variables becomes a problem for large $n$. This effect is shown in Figure 6: on the $x$ axis we take the angle $\theta$ to characterise an ejection orbit, on the $y$ axis the time it takes for such orbit to cross for the $2 n$-th time the Poincaré section $v=0$ (in McGehee variables). The small cusps on each curve correspond to very close approaches to collision, so that if the grid of $\theta$ values is refined the spike grows higher and tends to a vertical asymptote (infinite time to reach the collision). Each singularity (vertical asymptote) in time corresponds to an EC orbit and for the curve $\left(\theta_{0}, T_{2}\right)$ only four singularities appear, corresponding to the four 1-EC orbits. For the curve $\left(\theta_{0}, T_{4}\right)$ we observe 8 singularities: there are the previous existing 1-EC orbits and four new ones corresponding to the 2-EC orbits we are looking for. It is clear that for large $n$ it is really difficult to detect the new EC orbits and to distinguish them from any previous ones with a smaller $n$. We remark also the big intervals of time needed for the largest values of $n$. These drawbacks completely disappear when using Levi-Civita for the same simulation: one would see almost straight lines, due to the regular ODE, and the ranges of time are sensitively smaller.
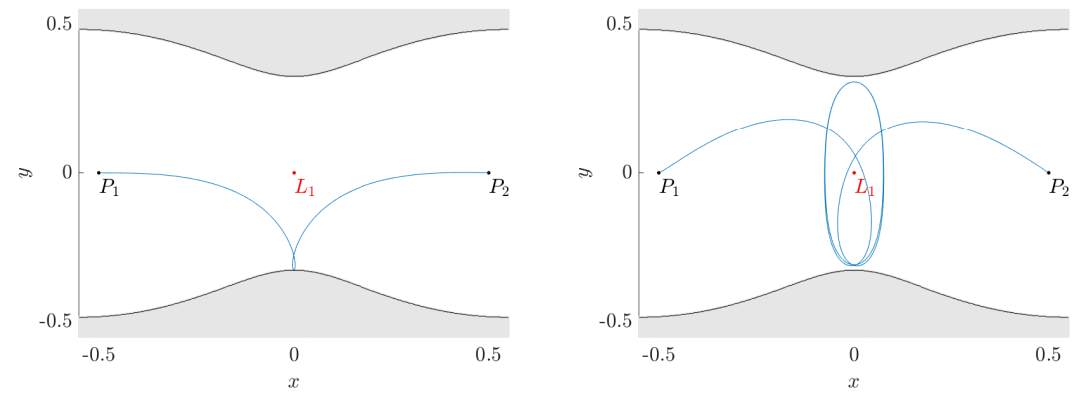

Fig. $7 \mu=0.5,(x, y)$ coordinates. An orbit ejecting from the big primary and colliding with the small one. Left. A direct trajectory. Right. A trajectory describing a turn around the Lyapunov PO.

Finally we remark that for higher values of $H$, say $H<H_{L_{2}}(\mu)$, the dynamics is richer due to two effects. The first one is that the bounded Hill region allows connections between both primaries. An example is shown in Figure 7 left, where an ejection orbit from the big primary collides with the small one. A second effect is due to the Lyapunov periodic orbit PO around $L_{1}$ and its stable and unstable manifolds, which play a role when going from the neighborhood of one primary to the neighborhood of the other one. In Figure 7 right we show an orbit which ejects from the big primary, describes a turn around the PO and collides with the small primary.

A detailed description of the variety of motions for higher values of $H$ (where the particle can leave a neighborhood of the primaries and even reach infinity) is to appear in a future paper. 


\section{Acknowledgments}

M. Ollé and O. Rodríguez were supported by the Spanish MINECO/FEDER grant MTM2015-65715-P and the Catalan grant 2017SGR-1049. J. Soler was supported by MINECO/FEDER grant number MTM2016-77278-P.

\section{References}

[1] Astakhov, S. A., Burbanks, A. D., Wiggins, S., Farrelly, D. Chaos-assisted capture of irregular moons. Lett. to Nature 2003;423:264-267.

[2] Astakhov, S. A., Lee, E. A., Farrelly, D. Formation of Kuiper-belt binaries through multiple chaotic scattering encounters with low-mass intruders. Mon. Not. R. Astron. Soc. 2005;360:401-415.

[3] Barrabés, E., Mondelo, J. M., Ollé, M. Numerical continuation of families of homoclinic connections of periodic orbits in the RTBP. Nonlinearity 2009;22:2901-2918.

[4] Bozis, G. Sets of collision periodic orbits in the Restricted problem. In: Periodic orbits, stability and resonances, G.E.O. Giacaglia (eds). Holland: D. Reidel Pub. Co.; 1970, p. 176-191.

[5] Brunello, A. F.,Uzer, T., Farrelly, D. Hydrogen atom in circularly polarized microwaves: Chaotic ionization via core scattering. Phys Rev A 1997;55:3730-3745.

[6] Chenciner, A and Llibre, J. A note on the existence of invariant punctured tori in the planar circular RTBP. Ergod. Th. \& Dynam. Sys. 1988;8:63-72.

[7] Devaney, R. L. Singularities in Classical Celestial Mechanics. In: Ergodic Theory and Dynamical Systems I, Proceedings Special year, Maryland 1979-80, A. Katok (Ed.), p. 211-333.

[8] Hénon, M. Exploration numérique du problème restreint I. Masses égales, Orbites périodiques. Ann Astrophys 1965;28:499-511.

[9] Hénon, M. Numerical exploration of the Restricted Problem V. Hill's case: Periodic orbits and Their Stability. Astron Astrophys 1969;1:223-238.

[10] Lacomba, E. A. and Llibre, J. Transversal Ejection-Collision Orbits for the Restricted Problem and the Hill's Problem with Applications. J Differ Eq 1988;74:69-85.

[11] Llibre, J. On the Restricted Three-Body Problem when the Mass Parameter is Small. Celest Mech Dyn Astron 1982;28:83-105.

[12] Llibre, J, and Martinez-Alfaro, J. Ejection and collision orbits of the spatial RTBP. Celest Mech Dyn Astron 1985;35:113-128.

[13] Llibre, J. and Pinyol, C. On the Elliptic Restricted Three-Body Problem. Celest Mech Dyn Astron 1990;48:319-345.

[14] McGehee, R. Triple Collision in the Collinear Three-Body Problem. Invent Math 1974;27:191-227.

[15] Ollé, M. To and fro motion for the hydrogen atom in a circularly polarized microwave field. Commun Nonlinear Sci Numer Simulat 2018;54:286-301.

[16] Ollé, M.; Rodríguez, O.; Soler, J. Ejection-collision orbits in the RTBP. Commun Nonlinear Sci Numer Simulat 2017;55:298-315.

[17] Pinyol, C. Ejection-collision orbits with the more massive primary in the planar elliptic restricted three-body problem. Celest Mech Dyn Astron 1995;61:315-331.

[18] Rodríguez, O. Òrbites d'ejecció-col.lisió en el problema restringit de tres cossos. Master Thesis. Universitat Politècnica de Catalunya, 2016.

[19] Sanchez, D.P., and Scheeres, D.J. DEM simulation of rotation-induced reshaping and disruption of rubble-pile asteroids. Icarus, 218, 876-894 (2012).

[20] Stiefel, E. L., Scheifele, G. Linear and regular Celestial Mechanics. Springer-verlag, New York; 1971.

[21] Szebehely, V. Theory of orbits. Academy Press, Inc., New York; 1967. 